BMJ Open

Diabetes

Research

\& Care

\section{Habitual snoring, adiposity measures and risk of type 2 diabetes in 0.5 million Chinese adults: a 10-year cohort}

To cite: Wei Y, Zheng B, Fan J, et al. Habitual snoring, adiposity measures and risk of type 2 diabetes in 0.5 million Chinese adults: a 10 -year cohort. BMJ Open Diab Res Care 2020;8:e001015. doi:10.1136/ bmjdrc-2019-001015

- Additional material is published online only. To view please visit the journal online (http://dx.doi.org/10.1136/ bmjdrc-2019-001015).

YW and BZ are joint first authors.

Received 30 0ctober 2019 Revised 31 December 2019 Accepted 14 January 2020
Check for updates

(C) Author(s) (or their employer(s)) 2020. Re-use permitted under CC BY-NC. No commercial re-use. See rights and permissions. Published by BMJ.

For numbered affiliations see end of article.

Correspondence to Dr Canqing Yu; yucanqing@pku.edu.cn and Professor Liming Li; Imlee@vip.163.com

\section{ABSTRACT}

Objectives The present study aimed to examine whether habitual snoring was independently associated with risk of type 2 diabetes among Chinese adults, and to assess the role that adiposity measures play in the snoring-diabetes association, as well as to evaluate the joint influence of snoring and adiposity measures on diabetes.

Research design and methods The China Kadoorie Biobank study recruited 512715 adults aged 30-79 years from 10 regions in China during 2004 and 2008. Data from 482413 participants without baseline diabetes were analyzed in the present study. Autoregressive crosslagged panel analysis was used to assess the longitudinal relationship between adiposity measures and habitual snoring. Cox proportional hazards models were used to examine the association between habitual snoring and diabetes risk.

Results Both higher body mass index and waist circumference were associated with higher risks of subsequent habitual snoring, whereas no reverse association was detected. A total of 16479 type 2 diabetes cases were observed during a 10-year follow-up. Habitual snoring was independently associated with $12 \%(95 \% \mathrm{Cl}$ $6 \%$ to $18 \%$ ) and $14 \%$ (95\% Cl $9 \%$ to $19 \%$ ) higher risks of diabetes among men and women, respectively. Habitual snorers who had general obesity or central obesity were about twice as likely to develop diabetes as non-snorers at the lowest levels of adiposity measures.

Conclusion Habitual snoring was independently associated with a higher risk of type 2 diabetes among Chinese adults. It is important to maintain both a healthy weight and a normal waist circumference to prevent or alleviate habitual snoring and ultimately prevent diabetes among Chinese adults.

\section{INTRODUCTION}

Diabetes now is affecting more than 475 million people and has become the fourth leading cause of disability globally. ${ }^{1}$ The estimated standardized prevalence of diabetes was as high as $10.9 \%$ among Chinese adults in $2013 .^{2}$ Snoring is the most common symptom of obstructive sleep apnea (OSA) characterized by repetitive episodes of complete or partial upper airway obstruction

\section{Significance of this study}

What is already known about this subject?

- Previous studies from mostly Western countries suggested that habitual snoring was associated with increased risks of type 2 diabetes.

- High-quality evidence on snoring-diabetes association is lacking for the Asian population whose adiposity characteristics are different from those of the Western population.

What are the new findings?

- Both higher body mass index and waist circumference were associated with higher risks of subsequent habitual snoring.

- Habitual snoring was independently associated with an increased risk of type 2 diabetes among Chinese adults.

- Habitual snorers who had general obesity or central obesity were about twice as likely to develop diabetes as non-snorers at the lowest levels of adiposity measures.

How might these results change the focus of research or clinical practice?

- These findings demonstrate that it is important to maintain both a healthy weight and a normal waist circumference to prevent or alleviate habitual snoring and ultimately prevent diabetes among Chinese adults.

occurring during sleep. ${ }^{3-5}$ The prevalence of habitual snoring ranges from $13.6 \%$ to $31.4 \%$ among men and from $4.3 \%$ to $16.2 \%$ among women in different studies in the Asian population. ${ }^{6-10}$

Previous studies suggested that habitual snoring and OSA increased the risk of type 2 diabetes. ${ }^{11-15}$ As the information on snoring status is more convenient to collect in largescale research or population screenings compared with a clinical diagnosis of OSA with objective measurements, estimating the association between snoring and diabetes has 
more public health implications and can help identify individuals at higher risks of type 2 diabetes. However, previous snoring-related studies are mostly conducted in the Western population, ${ }^{11-14}$ and high-quality evidence is lacking for the Asian population with different adiposity characteristics. ${ }^{16}$ Some cross-sectional studies found a positive correlation between habitual snoring and the worsening of glucose metabolism indexes in Asian adults. ${ }^{18-22}$

In the present study, we use data from the China Kadoorie Biobank (CKB), a nationwide prospective study conducted among half a million Chinese adults aged 30-79 years, to examine whether habitual snoring was independently associated with a higher risk of type 2 diabetes among the Chinese population, and to explore the mediating or confounding effect of adiposity measures on the snoring-diabetes association, as well as the joint impact of habitual snoring and different adiposity measures on the risk of diabetes.

\section{RESEARCH DESIGN AND METHODS}

\section{Study population}

The baseline survey of the CKB study was conducted in five urban and five rural areas across China from 2004 to 2008. The cohort enrolled a total of 512715 participants aged 30-79 years who had complete baseline information, including a questionnaire, anthropometric measurements, blood sample collection, and a written informed consent. About $5 \%$ of randomly chosen surviving participants were resurveyed twice with the same measure as in the baseline. The first resurvey was conducted in 2008 and the second one between 2013 and 2014. More details about the study design are available in previous publications. ${ }^{23} 24$

To analyze the association between habitual snoring and risk of type 2 diabetes, we excluded participants who had previously physician-diagnosed or screen-detected diabetes at baseline $(\mathrm{n}=30300)$, lacked information on baseline body mass index (BMI, $\mathrm{n}=2$ ), or lost to follow-up immediately after they completed the baseline survey $(n=1)$, and thereby included a total of 482413 participants.

\section{Data collection}

In the baseline questionnaire, participants were asked whether they snored during sleep, and the options were: (1) Yes, often; (2) Yes, sometimes; (3) Never/I do not know. In the present analysis, participants with the first option were categorized as habitual snorers while the rest of all were non-snorers. ${ }^{25}{ }^{26}$ According to the quality control study done in 15718 participants at a median interval of 7 days (IQR: 3-15 days) after the baseline survey, the kappa value for repeatability of habitual snoring was 0.657 . Information about other sleep characteristics such as total sleep duration (including the duration of daytime napping) and daytime napping frequency (always, only in summer, or never) was also collected at baseline.

The baseline survey also collected information about sociodemographic characteristics (age, gender, educational attainment, household income, and marital status), lifestyle factors (alcohol intake, smoking status, physical activity, and consumption of fresh vegetables, fresh fruit, and red meat), self-reported diagnosis of chronic diseases (hypertension, diabetes, stroke or transient ischemic attack, and coronary heart disease), family history of diabetes, and menopausal status (only for women).

For each participant, a $10 \mathrm{~mL}$ non-fasting blood sample (with the time of last meal recorded) was collected into one EDTA vacutainer (BD Hemogard, USA). ${ }^{23}$ For participants with a random blood glucose level $\geq 7.8$ and $<11.1 \mathrm{mmol} / \mathrm{L}$, a fasting blood glucose test was done the following day. The screen-detected diabetes was defined as a random glucose level $\geq 11.1 \mathrm{mmol} / \mathrm{L}$ or a fasting glucose level $\geq 7.0 \mathrm{mmol} / \mathrm{L}$, but without self-reported diabetes.

\section{Anthropometric measurements}

Trained health workers measured standing height, body weight, and waist circumference of participants using calibrated instruments following standard procedures. Participants were measured for standing height in bare feet and body weight in light clothes. BMI was calculated using the following formula: BMI $\left(\mathrm{kg} / \mathrm{m}^{2}\right)=$ weight $(\mathrm{kg}) /$ (height $(\mathrm{m}))^{2}$. BMI was grouped into three categories according to Chinese guidelines, ${ }^{27}$ namely underweight/ normal for BMI $<24.0 \mathrm{~kg} / \mathrm{m}^{2}$, overweight for BMI 24.0 $27.9 \mathrm{~kg} / \mathrm{m}^{2}$, and general obesity for BMI $\geq 28.0 \mathrm{~kg} / \mathrm{m}^{2}$. Waist circumference was also grouped into three categories (not central obesity: waist circumference $<85 \mathrm{~cm}$ for men and $<80 \mathrm{~cm}$ for women, precentral obesity: waist circumference $85.0-89.9 \mathrm{~cm}$ for men and $80.0-84.9 \mathrm{~cm}$ for women, central obesity: waist circumference $\geq 90 \mathrm{~cm}$ for men and $\geq 85 \mathrm{~cm}$ for women) according to Chinese guideline. ${ }^{27}$

\section{Identification of incident type 2 diabetes}

The CKB study followed up participants from the time they completed baseline questionnaires until the occurrence of type 2 diabetes, death, loss to follow-up, or 31 December 2016, whichever came first. Type 2 diabetes cases were ascertained through linkage to the disease surveillance system, death registries, and the national health insurance system, and through active follow-up. Participants were identified in these systems with their Chinese resident identity card numbers. Trained staff who were blinded to baseline information of participants coded the type 2 diabetes cases according to the International Classification of Diseases, 10th Version . In the present study, type 2 diabetes was defined using the codes of E11 (type 2) and E14 (unspecific) but not E10 (type 1) from the sources mentioned earlier during the follow-up to minimize under-reporting of type 2 diabetes. 


\section{Statistical analysis}

The baseline characteristics of participants with different snoring status were presented as means and percentages adjusted for age, gender, and study region via general linear regression for continuous variables and logistic regression for categorical variables.

\section{Bidirectional analysis of adiposity measures and habitual snoring}

An autoregressive cross-lagged panel analysis was conducted among a subcohort of 25037 participants participating in both the baseline and second resurvey to assess the longitudinal relationship between BMI, waist circumference, and habitual snoring. The generalized structural equation model was used, with BMI, waist circumference, and habitual snoring at second resurvey as the outcomes and their baseline values as the independent variables, adjusting for baseline characteristics including age, gender, study region, educational attainment, household income, marital status, smoking status, alcohol consumption, and physical activity level.

\section{Assessment of the association between habitual snoring and} diabetes

Among the 482413 participants, Cox proportional hazards models were used to estimate HRs of type 2 diabetes for habitual snorers in comparison with nonsnorers among men and women, respectively. Using age at follow-up as the timescale, the Cox models were stratified by age at baseline (in 5-year interval) and 10 study regions, and were adjusted for a range of baseline factors, including education, household income, marital status, BMI, waist circumference, smoking status, alcohol intake, physical activity, family history of diabetes, prevalent hypertension, prevalent stroke or transient ischemic attack, prevalent coronary heart disease, menopausal status (only for women), total sleep duration, daytime napping, and dietary factors. The method of Schoenfeld residuals found no violation of the proportional hazards assumption for habitual snoring in the fully adjusted model.

Several subgroup analyses were further conducted according to participants' BMI, waist circumference, and other baseline factors. Multiplicative interactions between habitual snoring and these baseline factors were assessed with likelihood ratio tests. In addition, we performed joint analyses to estimate the relative risks of type 2 diabetes for participants with different combinations of snoring status and BMI or waist circumference, with non-snorers at the lowest levels of BMI or waist circumference treated as the reference groups.

Sensitivity analyses were conducted by additionally adjusting for body fat percentage, or excluding type 2 diabetes events happening during the first 2 years of follow-up to account for reverse causality, or by excluding participants who were never married, divorced or widowed at baseline to minimize under-reporting of snoring symptom. Besides, to explore the potential impact of the underdiagnosis of type 2 diabetes during the follow-up, we have updated incident diabetes cases in a subcohort of participants using information about self-reported and screen-detected diabetes at the second resurvey and assessed the snoring-diabetes association in this subcohort of participants. To assess whether occasional snoring was associated with risk of type 2 diabetes, 'sometimes snorers' were also compared with never snorers in multivariable-adjusted Cox proportional hazards models.

For primary analyses and sensitivity analyses, statistical significance was defined as $\mathrm{p}<0.05$. For subgroup analyses and multiplicative interaction tests, the Bonferroni correction was used, and the statistical significance was defined as $\mathrm{p}<0.003$ (16 subgroup analyses were conducted). All statistical analyses were carried out using Stata V.15.0 (StataCorp, College Station, Texas, USA).

\section{RESULTS}

Among the 482413 participants, $28.3 \%$ of male participants and $16.7 \%$ of female participants were habitual snorers. Habitual snorers were more likely to be older, urban residents, current daily smokers, current weekly alcohol consumers, regular red meat consumers, to have higher adiposity measures, and to take a nap in the daytime $(p<0.05)$. They also tended to have a family history of diabetes, a diagnosis of hypertension, stroke or transient ischemic attack, and coronary heart disease at baseline. Among male participants, habitual snorers were also more likely to be married and with higher family income (table 1).

The ORs of habitual snoring at second resurvey were 1.31 (95\% CI 1.23 to 1.40$)$ and 1.10 (95\% CI 1.03 to 1.17$)$ for per SD increment in baseline BMI and waist circumference, but no significant association was observed for habitual snoring at baseline with neither BMI ( $\beta$ : $-0.023,95 \% \mathrm{CI}-0.075$ to 0.028$)$ nor waist circumference ( $\beta$ : $0.018,95 \% \mathrm{CI}-0.166$ to 0.201 ) at second resurvey (figure 1 and online supplementary table e-1).

A total of 16479 incident cases of type 2 diabetes were identified during a median follow-up of 10.1 years among 482413 participants. Habitual snorers were 1.47 (95\% CI 1.39 to 1.54$)$ and 1.48 (95\% CI 1.41 to 1.52$)$ times more likely to develop type 2 diabetes than non-snorers among men and women in models adjusted for baseline factors except for adiposity measures (table 2). The association was markedly attenuated with further adjustment for BMI and waist circumference. However, habitual snoring was still independently associated with $12 \%$ (95\% CI $6 \%$ to $18 \%$ ) and $14 \%$ (95\% CI $9 \%$ to $19 \%)$ higher risks of type 2 diabetes after full adjustment among men and women, respectively ( $p$ value for interaction $=0.320$ ).

Subgroup analyses showed that the association between habitual snoring and type 2 diabetes remained consistent within each stratum of adiposity measures and other baseline characteristics (figure 2, online supplementary figure e-1). No multiplicative interaction was found between snoring status and adiposity measures 
Table 1 The baseline characteristics of participants according to snoring status among 482413 participants

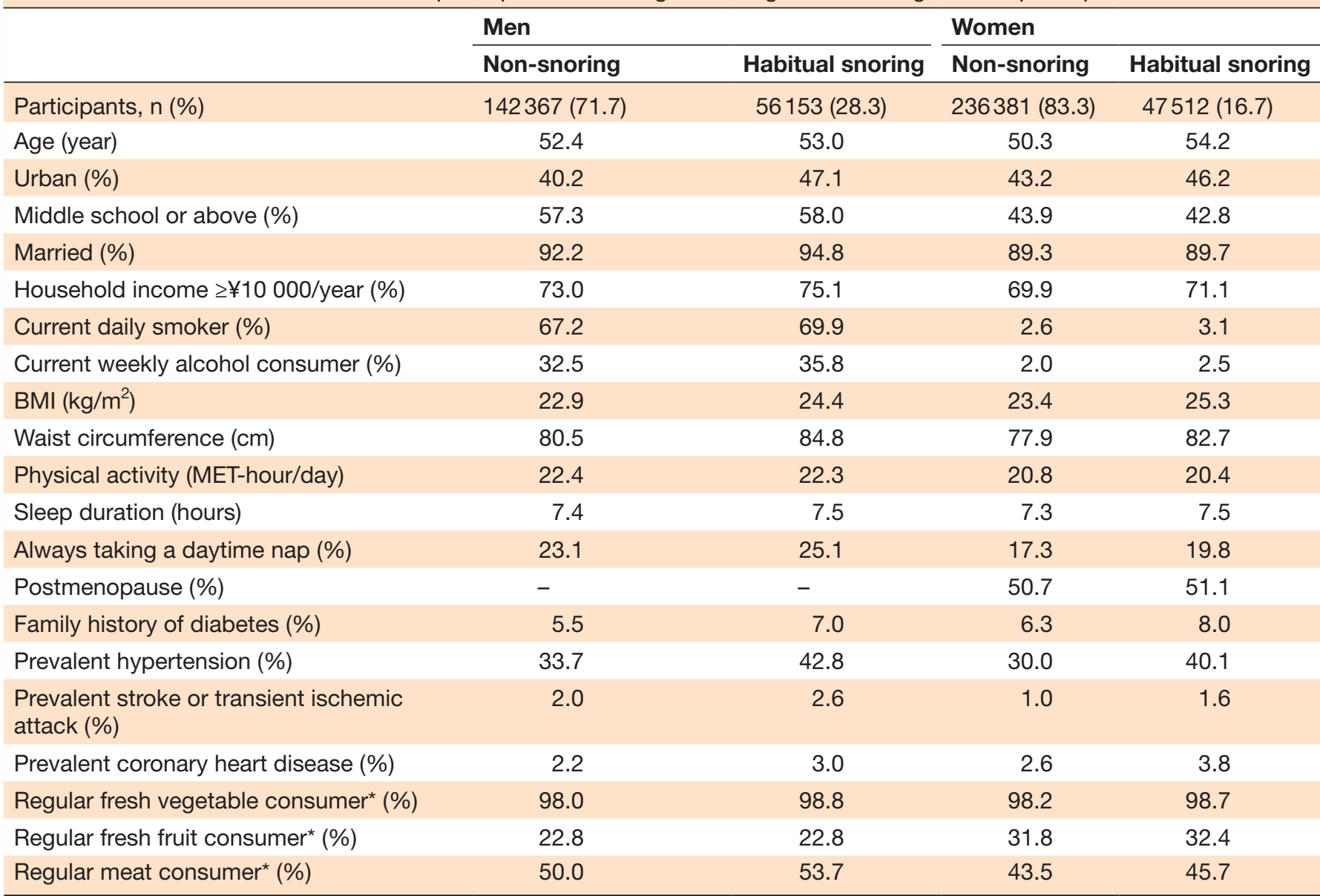

Values of variables except age and urban were adjusted for age at study date and study regions, when appropriate.

${ }^{*}$ Regular consumers were referred to those consuming corresponding foods at least four times a week.

BMI, body mass index; MET, metabolic equivalent.

or other baseline characteristics. Sensitivity analyses showed no major changes in risk estimates by additionally adjusting for body fat percentage, excluding diabetes cases identified during the first 2 years of follow-up, or excluding participants who were never married, divorced

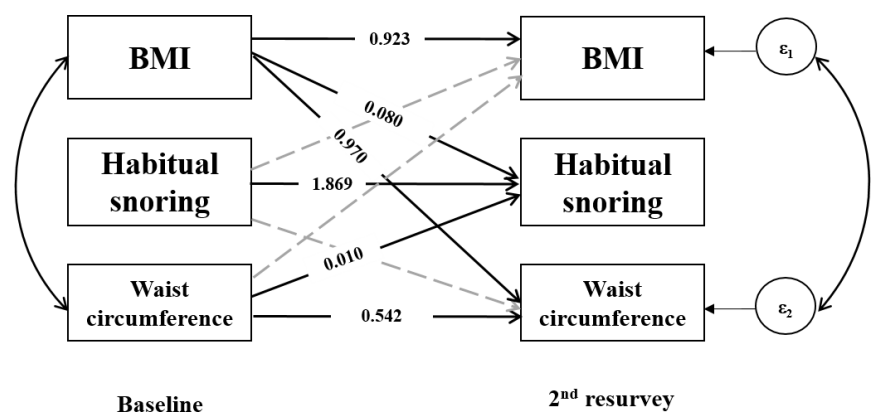

Figure 1 The autoregressive cross-lagged model between $\mathrm{BMI}\left(\mathrm{kg} / \mathrm{m}^{2}\right)$, waist circumference $(\mathrm{cm})$, and habitual snoring. The model was adjusted for baseline characteristics including age, gender, study region, educational attainment, household income, marital status, smoking status, alcohol consumption, and physical activity level. Solid lines show coefficients with $p$ values $\leq 0.05$ while dashed lines present coefficients with $p$ values $>0.05$. BMI, body mass index. or widowed at baseline (online supplementary table e-2). The sensitivity analysis conducted among a subcohort of participants with updated information about incident diabetes at the second resurvey also showed a similar risk estimate (HR: $1.17,95 \%$ CI 1.05 to 1.31 ; online supplementary table e-3) to the primary analysis. In addition, occasional snoring was associated with a minor increased risk of type 2 diabetes among women (HR: 1.07, 95\% CI 1.02 to 1.12 ) but not among men (HR: $0.94,95 \%$ CI 0.88 to 1.00; online supplementary table e-4) when compared with never snorers.

Compared with non-snorers who were underweight or normal weight, the relative risks of diabetes for general obese non-snorers were 1.80 (95\% CI 1.57 to 2.05) among men and 1.65 (95\% CI 1.51 to 1.80 ) among women, which rose to 2.06 (95\% CI 1.81 to 2.34) among men and 1.83 (95\% CI 1.66 to 2.02) among women for general obese habitual snorers. Similarly, in the joint analysis of snoring and central obesity, participants who were both central obese and habitual snorers had the highest risk of type 2 diabetes, which was 1.96 (95\% CI 1.77 to 2.18) for men and 1.97 (95\% CI 1.82 to 2.14) for women (figure 3, online supplementary table e-5). 
Table 2 The association between habitual snoring and type 2 diabetes among 482413 participants

\begin{tabular}{|c|c|c|c|c|c|}
\hline & \multicolumn{2}{|l|}{ Men } & \multicolumn{2}{|l|}{ Women } & \multirow{2}{*}{$\begin{array}{l}P \text { value for } \\
\text { interaction* }\end{array}$} \\
\hline & Non- snoring & Habitual snoring & Non- snoring & Habitual snoring & \\
\hline Cases & 3700 & 2611 & 7271 & 2897 & \\
\hline Person-years & 1376688 & 542611 & 2357347 & 464937 & \\
\hline $\begin{array}{l}\text { Incidence rate (per } 10000 \\
\text { person-years) }\end{array}$ & 26.9 & 48.1 & 30.8 & 62.3 & \\
\hline Model 1 & 1.00 & $1.59(1.51,1.67)$ & 1.00 & $1.62(1.55,1.69)$ & 0.192 \\
\hline Model 2 & 1.00 & $1.58(1.50,1.66)$ & 1.00 & $1.62(1.55,1.69)$ & 0.206 \\
\hline Model 3 & 1.00 & $1.48(1.40,1.55)$ & 1.00 & $1.49(1.42,1.56)$ & 0.417 \\
\hline Model 4 & 1.00 & $1.47(1.39,1.54)$ & 1.00 & $1.48(1.41,1.52)$ & 0.441 \\
\hline Model 5 & 1.00 & $1.12(1.06,1.18)$ & 1.00 & $1.14(1.09,1.19)$ & 0.320 \\
\hline
\end{tabular}

Model 1: Stratified by age groups and 10 study regions. Adjusted for education, household income, and marital status.

Model 2: Additionally adjusted for smoking status, alcohol intake, and physical activity based on model 1.

Model 3: Additionally adjusted for family history of diabetes, prevalent hypertension, prevalent stroke or transient ischemic attack, and prevalent coronary heart disease based on model 2.

Model 4: Additionally adjusted for menopausal status (only for women), sleep duration, daytime napping, fresh vegetables, fresh fruit, and red meat consumption based on model 3.

Model 5: Additionally adjusted for baseline body mass index (BMI) and baseline waist circumference based on model 4 .

${ }^{*} \mathrm{P}$ values for multiplicative interaction between habitual snoring and gender.

\section{DISCUSSION}

The present study, prospectively conducted among a nationwide sample of 0.5 million Chinese adults, indicated that adiposity measures were associated with an increased risk of future habitual snoring, and the latter was associated with a higher risk of type 2 diabetes in both men and women independent of adiposity measures. Besides, participants who had both habitual snoring and

Subgroup

Cases*/person years

$\mathrm{HR}(95 \% \mathrm{Cl})$

$P$ values for interaction

Men

BMI subgroups

Underweight/normal

Overweight

General obesity

$2,120 / 1,137,346$
$2,843 / 620,664$
$1,348 / 161,289$

$1.10(1.00,1.21)$

0.924

Waist circumference subgroups

Not central obesity

Pre-central obesity

Central obesity

$2,447 / 1,218,498$
$1,249 / 307,528$
$2,615 / 393,273$

$1.12(1.03,1.20)$

$1.16(1.04,1.30)$

$1.10(1.01,1.21)$

$1.20(1.07,1.35)$

$1.09(1.01,1.19)$

Women
BMI subgroups
Underweight/normal
Overweight
General obesity
Waist circumferenc
Not central obesity
Pre-central obesity
Central obesity

$3,358 / 1,582,798$
$4,294 / 930,584$
$2,516 / 308,902$

$3,353 / 1,637,302$
$2,200 / 518,252$
$4,615 / 666730$

$1.14(1.04,1.25)$

$1.15(1.07,1.23)$

$1.14(1.05,1.24)$

$1.16(1.06,1.27)$

$1.13(1.03,1.25)$

$1.13(1.07,1.21)$

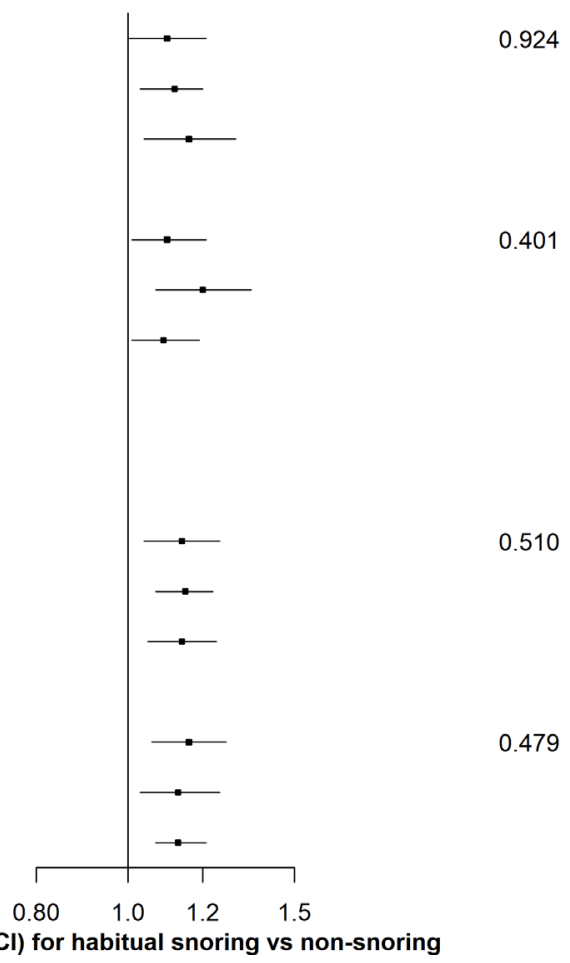

Figure 2 Subgroup analyses of the association between habitual snoring and type 2 diabetes according to different adiposity measures. ${ }^{*}$ Total incident diabetes cases for participants with habitual snoring and non-snoring. Models were stratified by age and 10 study regions, and adjusted for the same factors as in model 5 of table 2, except the subgrouping variables. BMI, body mass index. 

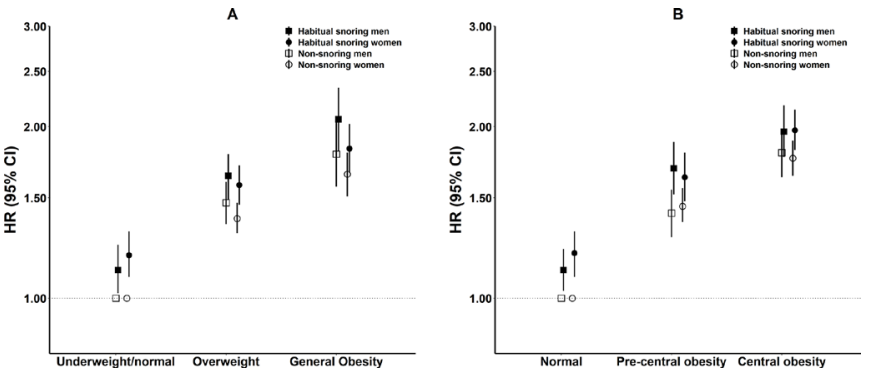

Figure 3 Adjusted HRs of diabetes for participants with different combinations of snoring status and adiposity measures. (A) Combination of snoring status and BMI subgroups. (B) Combination of snoring status and waist circumference subgroups. The Cox proportional hazards models were stratified by age and study region and were adjusted for the same factors as in model 5 of table 2, except baseline BMI and waist circumference. Baseline BMI was adjusted for in joint analyses of waist circumference and snoring while baseline waist circumference was adjusted for in joint analyses of BMI and snoring. BMI, body mass index.

general/central obesity were at the highest risk of developing type 2 diabetes, indicating a multiplicative joint effect.

A systematic review and meta-analysis involving eight studies from Western countries suggested that habitual snoring was associated with an increased risk of type 2 diabetes compared with non-snores, with a pooled OR of 1.37 (95\% CI 1.20 to 1.57$)$ for six cross-sectional studies and a pooled HR of 1.65 (95\% CI 1.30 to 2.08) for two cohorts. ${ }^{12}$ Mixed results from cohort studies in the Western population were reported. Two cohorts with small sample sizes in Sweden and the USA failed to spot a statistically significant association between snoring and diabetes. ${ }^{1125}$ The Nurses' Health Study (NHS) found that the relative risk of diabetes was 2.03 (95\% CI 1.71 to 2.40 ) for women who snored regularly in comparison with those who did not. ${ }^{28}$ Recently, a study on the NHS study and the Health Professionals Follow-up Study involving a total of 151194 participants suggested that habitual snoring without self-reported OSA was still independently associated with an increased risk of diabetes, although to a lesser extent (HR: $1.25,95 \%$ CI 1.19 to 1.31$).{ }^{14}$ Another prospective study conducted among 10854 participants from Northern Europe also found that snoring without self-reported OSA was independently associated with a higher risk of diabetes during a mean follow-up of 11.3 years. ${ }^{13}$

In contrast, prospective evidence is limited for the Asian population whose body sizes are different from their Western counterparts. ${ }^{16} 17$ Some cross-sectional studies suggested a positive correlation between habitual snoring and elevated glucose or hemoglobin A1c levels among Asian adults without prevalent diabetes. ${ }^{18}$ 20-22 However, cross-sectional studies are prone to reverse causation and other biases. The present study, which was the first largescale prospective cohort to assess the snoring-diabetes association among Chinese adults, found that habitual snoring was independently associated with a >10\% increased risk of type 2 diabetes.

The mechanisms through which habitual snoring increases the risk of type 2 diabetes remained unclear but might be attributed to obstruction of the upper airway during sleep, similar to the mechanism of OSA, as snoring without OSA was considered an early stage in the spectrum of sleep-disordered breathing. ${ }^{29-31}$ Hypoxia caused by upper airway obstruction promotes sympathetic activation and thereby disrupting insulin sensitivity and glucose tolerance, leading to an increased risk of type 2 diabetes. ${ }^{32-34}$

Previous cross-sectional studies observed a positive correlation between central/general obesity and habitual snoring among the Chinese population.7 212235 The present autoregressive cross-lagged analysis showed that the risks of habitual snoring within 8 years increased by $\sim 30 \%$ per SD increment in baseline BMI and by $10 \%$ per SD increment in baseline waist circumference. Therefore, adiposity measures were critical confounding factors and should be carefully controlled for when assessing the snoring-diabetes association, as adiposity measures were also associated with risk of diabetes. ${ }^{33-35}$ In the present study, the observed effects of snoring and obesity on diabetes risk followed a multiplicative scale, and habitual snorers who had general obesity or central obesity were at the highest risk of developing type 2 diabetes among all combinations of snoring and obesity status. In particular, although Chinese adults' mean BMI was lower than the Western population, they were more likely to be central obese than their Western counterparts at given BMI. ${ }^{16} 17$ Therefore, in the prevention of diabetes among Chinese adults, attention should not be focused solely on BMI. Snoring status and indicators of both general and central obesity should all be taken into account instead. Another clinical implication of our study is that even the earlystage symptom of sleep-disordered breathing can independently increase the risk of diabetes, highlighting the need for early detection and intervention for snoring.

The strengths of the present study include the geographical diversity of the study population, prospective design, long-term follow-up, and the high validity of diabetes diagnosis (a medical record review for about 1000 incident cases of type 2 diabetes found a positive predictive value of $97 \%$ ). Also, the detailed information on adiposity measures and other baseline characteristics made it possible to minimize confounding bias by carefully adjusting for a range of potential confounding factors, and the large sample size enabled us to extensively explore the snoring-diabetes association within each subgroup of adiposity measures. Nevertheless, there are some limitations. First of all, the present study did not assess OSA objectively using polysomnography and thus was unable to examine whether the observed association between habitual snoring and type 2 diabetes was entirely due to the impact of OSA, which is a challenge that most snoring-related epidemiological studies confront. Second, for most participants, snoring status 
was self-reported only once at baseline. Some participants might under-report their habitual snoring status, especially for those who live alone, and snoring status could change during the follow-up. However, the misclassification was more likely to be non-differential and tended to underestimate the strength of association. Also, in the sensitivity analysis, we further excluded participants who were never married, divorced, or widowed to minimize the under-reporting for people without bed partners, which revealed similar results. Third, the underdiagnosis of incident diabetes is a prevalent problem in China ${ }^{2}$ which may affect our findings in the present study. However, the sensitivity analysis done in a subcohort of participants with updated information on self-reported or screen-detected diabetes at the second resurvey found no major change in the risk estimate. Last, the possibility of residual confounding bias could not be ruled out due to the observational nature of this study.

\section{CONCLUSIONS}

In conclusion, adiposity was positively associated with subsequent habitual snoring, which was shown to be a risk factor of type 2 diabetes independent of different adiposity measures among Chinese adults. The coexistence of habitual snoring and general/central obesity led to the highest risk of type 2 diabetes, which highlights the need for raising the public's awareness in snoring and maintaining a healthy weight and waist circumference to prevent type 2 diabetes among Chinese adults. More prospective studies are warranted to verify our findings.

\section{Author affiliations}

${ }^{1}$ Department of Epidemiology and Biostatistics, School of Public Health, Peking University, Beijing, China

${ }^{2}$ School of Public Health, Faculty of Medicine, Imperial College London, London, UK ${ }^{3}$ Key Laboratory of Molecular Cardiovascular Sciences (Peking University), Ministry of Education, Beijing, China

${ }^{4}$ Peking University Institute of Environmental Medicine, Beijing, China

${ }^{5}$ Chinese Academy of Medical Sciences, Beijing, China

${ }^{6}$ Medical Research Council Population Health Research Unit (MRC PHRU), Nuffield Department of Population Health, University of Oxford, Oxford, UK

${ }^{7}$ Clinical Trial Service Unit and Epidemiological Studies Unit (CTSU), Nuffield Department of Population Health, University of Oxford, Oxford, UK

${ }^{8}$ Public Health Department, Pengzhou People's Hospital, Pengzhou, China

${ }^{9} \mathrm{NCDs}$ Prevention and Control Department, Pengzhou CDC, Pengzhou, China

${ }^{10}$ China National Center for Food Safety Risk Assessment, Beijing, China

Acknowledgements The chief acknowledgement is to the participants, the project staff, and the China National Centre for Disease Control and Prevention (CDC) and its regional offices for assisting with the fieldwork. We thank Judith Mackay in Hong Kong; Yu Wang, Gonghuan Yang, Zhengfu Qiang, Lin Feng, Maigeng Zhou, Wenhua Zhao, and Yan Zhang in China CDC; Lingzhi Kong, Xiucheng Yu, and Kun Li in the Chinese Ministry of Health; and Sarah Clark, Martin Radley, Mike Hill, Hongchao Pan, and Jill Boreham in the CTSU, Oxford, for assisting with the design, planning, organization, and conduct of the study.

Collaborators The China Kadoorie Biobank Collaborative Group (group members were listed in the online supplementary material).

Contributors $\mathrm{CY}$ and $\mathrm{LL}$ conceived and designed the study. $\mathrm{ZC}$ and JunC, as the members of CKB steering committee, designed and supervised the conduct of the whole study, obtained funding, and together with JL, YG, ZB, HD, LY, YC, JiaC, and $\mathrm{XZ}$ acquired the data. YW and JF analyzed the data. BZ dealt with methodological issues. YW wrote the first draft of the manuscript. CY and LL contributed to the interpretation of the results and critical revision of the manuscript for valuable intellectual content. All authors reviewed the manuscript and revised it critically when necessary. All authors approved the final manuscript and agreed to be accountable for all aspects of the work in ensuring that questions related to the accuracy or integrity of any part of the work are appropriately investigated and resolved. $C Y$ and $L L$ are the guarantors of the study.

Funding This work was supported by the National Key R\&D Program of China (2016YFC0900500, 2016YFC0900501, 2016YFC0900504), the Natural Science Foundation of China (91846303), the UK Wellcome Trust (202922/Z/16/Z, 088158/Z/09/Z, 104085/Z/14/Z), and the Kadoorie Charitable Foundation in Hong Kong.

Disclaimer The funders had no role in the study design, data collection, data analysis and interpretation, writing of the report, or the decision to submit the article for publication.

Competing interests None declared.

Patient consent for publication Not required.

Ethics approval The CKB study was approved by the Ethical Review Committee of the Chinese Center for Disease Control and Prevention (Beijing, China) and the Oxford Tropical Research Ethics Committee, University of Oxford (UK).

Provenance and peer review Not commissioned; externally peer reviewed.

Data availability statement Data are available upon reasonable request. Details of how to access China Kadoorie Biobank data and details of the data release schedule are available on www.ckbiobank.org/site/Data+Access.

Open access This is an open access article distributed in accordance with the Creative Commons Attribution Non Commercial (CC BY-NC 4.0) license, which permits others to distribute, remix, adapt, build upon this work non-commercially, and license their derivative works on different terms, provided the original work is properly cited, appropriate credit is given, any changes made indicated, and the use is non-commercial. See: http://creativecommons.org/licenses/by-nc/4.0/.

ORCID iDs

Huaidong Du http://orcid.org/0000-0002-9814-0049

Canqing Yu http://orcid.org/0000-0002-0019-0014

\section{REFERENCES}

1 GBD 2017 Disease and Injury Incidence and Prevalence Collaborators. Global, regional, and national incidence, prevalence, and years lived with disability for 354 diseases and injuries for 195 countries and territories, 1990-2017: a systematic analysis for the global burden of disease study 2017. Lancet 2018;392:1789-858.

2 Wang L, Gao P, Zhang M, et al. Prevalence and ethnic pattern of diabetes and prediabetes in China in 2013. JAMA 2017;317:2515-23.

3 Whyte KF, Allen MB, Jeffrey AA, et al. Clinical features of the sleep apnoea/hypopnoea syndrome. Q J Med 1989;72:659-66.

4 Aldrich MS. Sleep medicine. New York: Oxford University Press, 1999.

5 Sateia MJ. International classification of sleep disorders-third edition: highlights and modifications. Chest 2014;146:1387-94.

6 Khoo SM, Tan WC, Ng TP, et al. Risk factors associated with habitual snoring and sleep-disordered breathing in a multi-ethnic Asian population: a population-based study. Respir Med 2004;98:557-66.

7 Liu J, Wei C, Huang L, et al. Prevalence of signs and symptoms suggestive of obstructive sleep apnea syndrome in Guangxi, China. Sleep Breath 2014:18:375-82.

8 Wali SO, Abaalkhail BA. Prevalence and predictors of habitual snoring in a sample of Saudi middle-aged adults. Saudi Med J 2015;36:920-7.

9 Ip MSM, Lam B, Tang LCH, et al. A community study of sleepdisordered breathing in middle-aged Chinese women in Hong Kong: prevalence and gender differences. Chest 2004;125:127-34.

10 Ip MS, Lam B, Lauder IJ, et al. A community study of sleepdisordered breathing in middle-aged Chinese men in Hong Kong. Chest 2001;119:62-9.

11 Strand LB, Carnethon M, Biggs ML, et al. Sleep disturbances and glucose metabolism in older adults: the cardiovascular health study. Diabetes Care 2015;38:2050-8.

12 Xiong X, Zhong A, Xu H, et al. Association between self-reported habitual snoring and diabetes mellitus: a systemic review and metaanalysis. J Diabetes Res 2016;2016:1958981.

13 Lindberg E, Benediktsdottir B, Franklin KA, et al. Women with symptoms of sleep-disordered breathing are less likely to be 
diagnosed and treated for sleep apnea than men. Sleep Med 2017;35:17-22.

14 Huang T, Lin BM, Stampfer MJ, et al. A population-based study of the bidirectional association between obstructive sleep apnea and type 2 diabetes in three prospective U.S. cohorts. Diabetes Care 2018;41:2111-9.

15 Nagayoshi M, Punjabi NM, Selvin E, et al. Obstructive sleep apnea and incident type 2 diabetes. Sleep Med 2016;25:156-61.

16 WHO Expert Consultation. Appropriate body-mass index for Asian populations and its implications for policy and intervention strategies. Lancet 2004;363:157-63.

17 NCD Risk Factor Collaboration (NCD-RisC). Trends in adult bodymass index in 200 countries from 1975 to 2014: a pooled analysis of 1698 population-based measurement studies with $19 \cdot 2$ million participants. Lancet 2016;387:1377-96.

18 Shin C, Kim J, Kim J, et al. Association of habitual snoring with glucose and insulin metabolism in nonobese Korean adult men. Am J Respir Crit Care Med 2005;171:287-91.

19 Lee YH, Kweon SS, Choi JS, et al. A gender-specific association between self-reported snoring and hemoglobin A1c levels in a general population without type 2 diabetes mellitus. Yonsei Med $J$ 2017;58:1152-9.

20 Wang H-B, Yan W-H, Dou J-T, et al. Association between selfreported snoring and prediabetes among adults aged 40 years and older without diabetes. Chin Med J 2017;130:791-7.

21 Huang J, Qi J, Lin Q, et al. Snoring and components of metabolic syndrome in southeastern Chinese adults: a community-based study. Clin Respir J 2018;12:966-73.

22 Li Y, Gao Q, Li L, et al. Additive interaction of snoring and body mass index on the prevalence of metabolic syndrome among Chinese coal mine employees: a cross-sectional study. BMC Endocr Disord 2019;19:28.
23 Chen Z, Chen J, Collins R, et al. China Kadoorie Biobank of 0.5 million people: survey methods, baseline characteristics and longterm follow-up. Int J Epidemiol 2011;40:1652-66.

24 Chen Z, Lee L, Chen J, et al. Cohort profile: the Kadoorie study of chronic disease in China (KSCDC). Int J Epidemiol 2005;34:1243-9.

25 Elmasry A, Janson C, Lindberg E, et al. The role of habitual snoring and obesity in the development of diabetes: a 10-year follow-up study in a male population. $J$ Intern Med 2000;248:13-20.

26 Valham F, Stegmayr B, Eriksson M, et al. Snoring and witnessed sleep apnea is related to diabetes mellitus in women. Sleep Med 2009;10:112-7.

27 National Health and Family Planning Commission of the People's Republic of China. Criteria of weight for adults. WS/T, 2013: 428-2013.

28 Al-Delaimy WK, Manson JE, Willett WC, et al. Snoring as a risk factor for type II diabetes mellitus: a prospective study. Am J Epidemiol 2002;155:387-93.

29 Young T, Palta M, Dempsey J, et al. The occurrence of sleepdisordered breathing among middle-aged adults. $N$ Engl $J$ Med 1993;328:1230-5.

30 Lugaresi E, Plazzi G. Heavy snorer disease: from snoring to the sleep apnea syndrome--an overview. Respiration 1997;64 Suppl 1:11-14.

31 Mohammadieh A, Sutherland K, Cistulli PA. Sleep disordered breathing: management update. Intern Med J 2017;47:1241-7.

32 Stamatakis KA, Punjabi NM. Effects of sleep fragmentation on glucose metabolism in normal subjects. Chest 2010;137:95-101.

33 Xie A, Skatrud JB, Puleo DS, et al. Exposure to hypoxia produces long-lasting sympathetic activation in humans. J Appl Physiol 2001;91:1555-62.

34 Nonogaki K. New insights into sympathetic regulation of glucose and fat metabolism. Diabetologia 2000;43:533-49.

35 Sun L, Pan A, Yu Z, et al. Snoring, inflammatory markers, adipokines and metabolic syndrome in apparently healthy Chinese. PLoS One 2011;6:e27515. 